\title{
Fundamental role of dendritic cells in inducing Th2 responses
}

\author{
Byoungjae $\mathrm{Kim}^{1,2}$ and Tae Hoon $\mathrm{Kim}^{1,3}$
}

${ }^{1}$ Department of Otorhinolaryngology-Head \& Neck Surgery, ${ }^{2}$ Neuroscience Research Institute, ${ }^{3}$ Allergy Immunology Center, Korea University College of Medicine, Seoul, Korea

Received: June 30, 2016

Accepted: September 5, 2017

\section{Correspondence to}

Tae Hoon Kim, M.D.

Department of Otorhinolaryn-

gology-Head \& Neck Surgery,

Korea University College of Medi-

cine, 73 Inchon-ro, Seongbuk-gu,

Seoul 02841, Korea

Tel: +82-2-920-5486

Fax: $+82-2-925-5233$

E-mail: doctorth@korea.ac.kr
A mysterious puzzle in immunology is how the immune system decides what types of immune response to initiate against various stimuli. Although much is known about control of T helper 1 (Th1) and Th17 responses, the mechanisms that initiate Th2 responses remain obscure. Antigen-presenting cells, particularly dendritic cells (DCs), are mandatory for the induction of a Th cell response. Numerous studies have documented the organizing role of DCs in this process. The present review summarizes the fundamental roles of DCs in inducing Th2 responses.

Keywords: Antigen-presenting cells; Dendritic cells; Th2 responses

\section{INTRODUCTION}

The immune system protects the host from pathogens, allergens, and chemicals that enter through mucosal surfaces. Diverse responses against these stimuli induce different types of immune response by various $\mathrm{T}$ helper (Th) cells. $\mathrm{CD}_{4}{ }^{+}$Th cells comprise a diverse category of immune cells, including Th1, Th2, Th17, and T regulatory cells. These different types of Th cell have distinct cytokines and transcription factor profiles with distinct immune responses. For example, $\mathrm{CD}_{4}+\mathrm{Th} 1$ responses are induced by intracellular bacteria and viruses, resulting in secretion of interferon $\gamma($ IFN- $\gamma$ ) and expression of signal transducer and activator of transcription (STAT)4 [1-3]. On the other hand, Th2 cells produce cytokines such as interleukin (IL)-4, IL-5, IL-13, and IL-10 and express transcription factors, such as GATA binding pro- tein-3, STAT-5, and STAT-6 [3-5].

Advances in immunology have revealed a fundamental role of the innate immune system in sensing pathogens and tuning the quality of Th responses. Thi cells attack intracellular pathogens with cell-mediated immune responses, whereas Th2 cells protect against extracellular pathogens predominantly by helping with humoral responses. When the Th1 and Th2 cell responses are overreactive, the Thr pathway induces organ-specific autoimmune diseases, such as arthritis and type 1 diabetes, whereas the Th2 pathway can predispose a host to systemic autoimmune diseases, such as allergy and atopic dermatitis $[6,7]$.

Differentiation of Th cells into Th1 or Th2 cells is under the control of antigen-presenting cells (APCs), mostly dendritic cells (DCs). When DCs are exposed to intracellular pathogens, they move to lymph nodes and 
secrete IL-12, resulting in differentiation of naïve T-cells into Thı cells. Those Thı cells release IFN- $\gamma$, which restimulates the DCs to produce IL-12, resulting in differentiation of naïve T-cells into Th1 through an autocrine loop. Like that of Th1 cells, maturation of Th2 cells is initiated by IL-6 released from DCs, and these Th2 cells produce IL-4 to generate more Th2 cells through an autocrine loop to naive T-cells. Several cell types produce cytokines to mature Th2 cells and induce Th2 immune responses. Diverse pattern recognition receptors (PRRs) are involved, and multiple signaling pathways are elicited (Fig. 1) [8]. However, the fundamental roles of DCs in the Th2 immune responses associated with these factors are not apparent. Thus, in this report we summarize previous studies of DC-related Th2 immune responses and present a new signaling pathway, the cAMP/protein kinase A (cAMP/PKA) signaling pathway, as a target for Th2 immune disease.

\section{INTERCELLULAR INTERACTIONS OF DENDRIT- IC CELLS IN TH2 IMMUNE RESPONSES}

A microbe entering the body can activate various immune cells, such as natural killer cells, basophils, mast cells, regulatory T-cells, and diverse structural cells, such as tissue epithelial cells and stromal cells. Upon activation by microbes, DCs orchestrate the complex action of these cell types to initiate and regulate the Th2 immune response.

Among various immune cells, basophils initiate IL-4

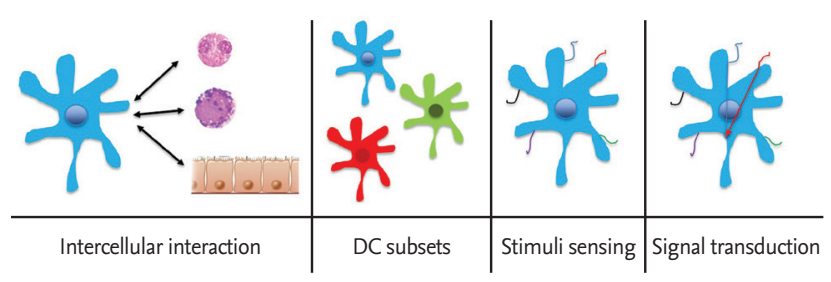

Figure 1. Levels that dendritic cells induce Th2 responses. For the induction of Th2 immune responses dendritic cells (DCs) require several steps in different levels of organization. Intercellular interaction between DC and other cells and specific DC subsets cause Th2 responses in upstream level of DC. In downstream level of DC, stimuli sensing and signal transduction of DCs are mandatory to induce Th2 responses. production during the memory $\mathrm{T}$-dependent response [9]. Furthermore, DCs or basophils alone are insufficient to induce the Th2 immune response in mice challenged by ovalbumin (OVA) plus papain, but cooperation between these two cell types allows DCs to induce T-cell proliferation and basophils to produce IL-4 for the Th2 immune response [10]. Therefore, the role of interaction between DCs and basophils in the induction of Th2 immune responses has been reviewed extensively [11-13]. However, the antigen-presenting role of basophils in Th2 immune responses described in these reviews is controversial because DCs are traditionally the major and sufficient APCs in Th2 immunity. Although basophils produce Th2 cytokines and are associated with induction of the Th2 immune response, whether they act as primary APCs in Th2 immunity is controversial. Taken together, data suggest that basophils behave as accessory cells to support DCs in inducing the Th2 immune response, in which DCs play a critical role as APCs and basophils produce Th2 cytokines, such as IL-4.

Innate lymphoid cells (ILCs) also orchestrate an efficient Th2 immune response in cooperation with tissueresident DCs. ILCs are classified as groups 1, 2 (ILC2), and 3 , which generate antigen-specific adaptive immune responses. In particular, ILC2s are a potent source of Th2 cytokines that promote Th2 immune responses [14]. ILC2s react to helminth infection in the intestine and allergens in the airways by producing Th2 cytokines, such as IL-5 and IL-13 [15,16]. In recent reports, ILC2-derived IL-13 stimulated lung DCs to migrate into draining lymph nodes, where they induced naïve T-cells to differentiate into Th2 cells and produce the chemokine CCL17 (C-C motif chemokine ligand 17), which attracts CCR4+ (C-C motif chemokine receptor 4+) memory Th2 cells $[17,18]$. These results suggest that DCs stimulated by ILC2s at the epithelial barrier are critical in Th2 immune responses.

In addition to immune cells, epithelial cells have been studied as major players in Th2 immune responses that influence DC functions, although the epithelium was initially regarded as a physical barrier [19]. DCs are always covered by epithelium, which causes the DC sentinel function and activation of DCs in the lungs [20]. The epithelial junction is dysregulated in cases of allergic inflammation, and these epithelial cells are susceptible to Th2 cytokines [21]. Epithelial cells also produce thymic 
stromal lymphopoietin (TSLP) during allergic inflammation, which subsequently activates CD11c+ DCs to induce Th2 immune responses [22].

\section{DENDRITIC CELL SUBSETS OF TH2 IMMUNE RESPONSES}

As APCs, DCs mediate immunity with several discrete cell subtypes, differentiated from lymphoid or myeloid precursor DCs. These different DC subtypes have a common antigen-presenting capacity to T-cells and promote cell-cycle progression. However, they activate Tcells differently, resulting in various Th responses [23]. For example, mouse $\mathrm{CD} 11 \mathrm{c}^{+} \mathrm{CD} 8 \alpha^{-} \mathrm{DCs}$ from the spleen induce a Th2 response, whereas CD $8 \alpha^{+}$DCs lead to Th1 differentiation [24]. In another murine experimental set, the lymphoid-related DC subset $\left(\mathrm{CD}_{11} \mathrm{c}^{+} \mathrm{CD} 8 \alpha^{+}\right.$ DEC205 $5^{+}$induced Thr cytokines, whereas the myeloid-

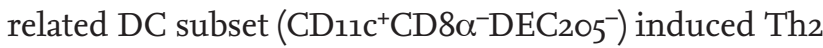
cytokines [25]. After OVA pulsing, DCs in the rat respiratory tract stimulated a Th2-dependent OVA-specific immunoglobulin (Ig) G1 response and mouse myeloid DCs induced Th2-dependent airway eosinophilia [26,27]. Mouse myeloid and plasmacytoid precursor DCs cultured from bone marrow precursors and ex vivo splenic DC subsets induced the development of Th1 or Th2 effector cells, depending on the antigen dose. In general, high antigen doses induce Thr cell development, whereas low antigen doses induce Th2 cell development [28]. $\mathrm{CD}_{3} \mathrm{O}^{\mathrm{b}}{ }^{+}$dermal DCs or bone marrow dendritic cells (BMDCs) drive the Th2 immune response in OT-II transgenic $\mathrm{CD}_{4}^{+} \mathrm{T}$-cells [29,30].

In humans, monocyte-derived DCs induce the Th immune response differentially, depending on the ratio of DCs to T-cells. At a low ratio (1:300), mature DCs induce the transformation of naïve T-cells into Th2 effector cells, whereas a high DC/T-cell ratio (1:4) favors mixed Th1/Th2 cell development [31]. Human monocyte-derived DCs induce the Th1 response, whereas CD11c ${ }^{-} \mathrm{CD}_{1} \mathrm{a}^{-}$plasmacytoid DCs favor the Th2 immune response [32,33]. In terms of tissues other than blood, epidermal $\mathrm{CD}_{2} \mathrm{O}^{+}$Langerhans cells preferentially induce $\mathrm{CD}_{4}{ }^{+} \mathrm{T}$-cells to secrete $\mathrm{Th} 2$ cell cytokines [34].

\section{STIMULUS SENSING AND PROCESSING OF DENDRITIC CELLS IN TH2 IMMUNE RESPONSES}

Activated DCs sense a diverse array of pathogens and allergens by PRRs, such as Toll-like receptors (TLRs), C-type lectin-like receptors (CLRs), RIG-I-like receptors, and Nod-like receptors, which are expressed in the surface and intracellular areas of DCs. Triggering of these PRRs activates DCs, leading to antigen-specific activation of Th cells [35,36]. The means by which microbial stimuli signal through PRRs to induce Thı immune responses are well understood, but our knowledge of the receptors that induce Th2 immune responses remains limited.

Among the PRRs on DCs, TLRs are the most studied. Various TLRs recognize microbial stimuli, such as lipopolysaccharide (LPS), lipoteichoic acids of Gram-positive bacteria and bacterial lipoproteins, and flagellin, and detect microbial nucleic acids, such as double-stranded RNA, single-stranded RNA, and CpG DNA. Although most TLR-eliciting signals induce Th1 responses, certain TLR ligands induce Th2 responses. Porphyromonas gingivalis LPS activates mouse DCs to produce Th2 cytokines through TLR4, whereas Escherichia coli LPS induces a Th1-like response [37]. The diacylated lipopeptide enhances production of Th2-type IgG1 antibodies in TLR2(+/+) mice, but not in TLR2(-/-) mice [38]. Stimulation of TLR2 with a synthetic TLR2 ligand elicits Th2 immune responses through extracellular regulated kinase (ERK) signaling in murine DCs [39]. In human DCs, TLR2 agonists yield a Th2 immune response, whereas triggering of TLR4 and TLR5 with E. coli LPS stimulates a Th1 response [40]. Activation of mouse DCs with a TLR2 ligand results in the induction of Th2 cytokines, such as IL-13, and promotes asthma in a mouse experimental model [41]. However, combined TLR2 and TLR4 activation by different antigens primes human DCs to induce Th1/Th2 immune responses [42]. Stimulation of human DCs by staphylococcal enterotoxin B through TLR2 drives naïve CD4 T-cells to develop a Th2 immune response [43]. Eosinophil-derived neurotoxin activates myeloid DCs by triggering the TLR2-myeloid differentiation factor 88 signaling pathway, which enhances an OVA-specific Th2 immune response [44]. In addition, TLR4 is necessary to induce Th2 responses to low-level LPS exposure in mouse DCs [45]. 
CLRs sense the carbohydrate regions of several pathogens and prime DCs to stimulate Th immune responses [46]. Dectins on mouse BMDCs and splenic DCs produce Th1 and Th17 cell responses, whereas DC-specific intercellular adhesion molecule-3-grabbing nonintegrin (DC-SIGN) induces a Th2 immune response on gastric DCs [47-49]. In addition, a ligand of DC-SIGN primes human DCs to induce the Th2 immune response [50].

Although PRR triggering of DCs is the main way to organize Th2 immune responses, other factors, such as the enzymes in allergens or alarmins from damaged tissue, can trigger Th2 immunity in the absence of PRR signaling [51]. IL-33 activates murine BMDCs to promote the Th2 immune response in allergic airway inflammation $[52,53]$. IL-25 also enhances the Th2 immune response of human TSLP-activated DCs [54].

\section{Dendritic cell signal transduction in Th2 immune responses}

Little is known about the signaling networks that stimulate DCs to induce a Th2 immune response. In contrast to LPS, which triggers TLR4 and induces DCs to initiate Th1 immune responses, the TLR2 ligand Pam3cys stimulates the duration and magnitude of the ERK mitogen-activated protein kinase in DCs and programs DCs to stimulate Th2 cell-biased responses [40]. These TLR2 ligands also elicit less IL-12p7o and more IL-10, consistent with other reports that Erk diminishes the induction of IL-12 and enhances IL-10 induction [55]. Furthermore, DCs from c-fos ${ }^{-/}$mice promote IL-12 production and negatively regulate IL-10 [39]. Taken together, these results indicate that the Erk-Fos signaling pathway is an important regulator of IL-12 and IL-10 production in DCs and promotes the Th2 immune response.

Another signaling pathway that induces DCs to initiate the Th2 immune response is nuclear factor- $\mathrm{B}(\mathrm{NF}-\kappa \mathrm{B})$. $\mathrm{NF}-\kappa \mathrm{B}$ is activated by TSLP and drives DCs to produce $\mathrm{OX}_{4} \mathrm{OL}$ and induce the Th2 immune response [56]. Th2 cytokines are not expressed in DCs of $\mathrm{NF}-\mathrm{\kappa BI}^{-/}$mice following injection of bacterial antigens [57]. Mouse BMDCs, stimulated by a helminth glycan, induce transient NF- $\mathrm{KB}$ translocation and activity in the nucleus and a Th2 immune response [58].

In addition to the above-described signaling pathways, knockout (KO) of Gnas, the gene that encodes Gas in mouse CDi1c+ cells, and the subsequent decrease of
cAMP in mouse DCs were recently reported to provoke the Th2 immune response with an allergic phenotype, whereas increased cAMP has been found to induce Th17 immunity (Fig. 2) [59,60]. In this study, adoptive transfer of BMDCs from the Gnas KO mice induced Th2 immune responses, such as increased IL-4 and elevated IgE, and a PKA-selective cAMP agonist eliminated the Th2 phenotype in these mice. These results indicate that the Th2-biased effects of low cAMP concentration in DCs are regulated via PKA signaling and that cAMP/ PKA signaling is an attractive target for the development of DC-directed therapy for Th2 immune diseases.

Further study of the role of the DC-related signaling pathway in Th2 immune responses is required to broaden the range of intracellular signaling pathways, some of which have not been anticipated previously.

\section{CONCLUSIONS}

DCs interact with multiple types of immune and structural cells, show distinct subsets, bind to specific receptors according to the pathogen or allergen, and transfer stimuli through diverse signaling pathways to elicit Th2 immune responses. Although DC-related Th2 immune responses are too complex to identify a single main

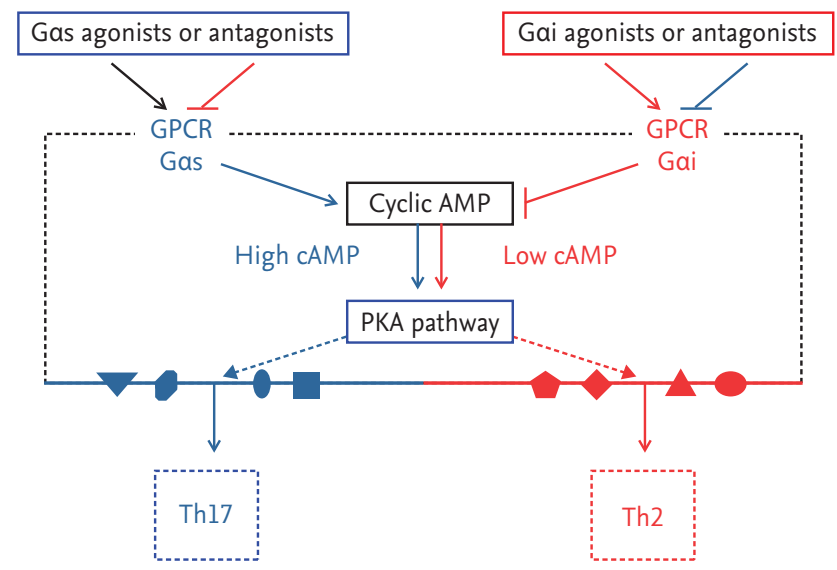

Figure 2. Novel signaling networks that provoke dendritic cell (DC) to induce Th2 or Th17 immunity. Decreased cyclic AMP (cAMP) concentration in dendritic cell provoke Th2 immunity via G-protein-coupled receptor (GPCR)/cAMP/ protein kinase A (PKA) signaling whereas increased cAMP concentration induce Th17 immunity through the same

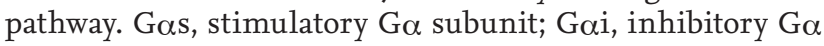
subunit. 
target factor and many aspects have not been revealed, newly discovered areas, such as cAMP/PKA signaling, illuminate the possibility of regulating Th2 immunity in the near future.

\section{Conflict of interest}

No potential conflict of interest relevant to this article was reported.

\section{Acknowledgments}

This research was supported by the Basic Science Research Program through the National Research Foundation of Korea funded by the Ministry of Science and Technology and the Ministry of Science, ICT \& Future Planning (2014R1A1A1002131, NRF-2016R1E1A2020731, 2017R1A2B2003575) and the Korea Health Technology R\&D Project ( $\mathrm{HI}_{17} \mathrm{Co}_{387}$ ) through the Korea Health Industry Development Institute (KHIDI) by the Ministry of Health and Welfare. This research was also supported by a Korea University grant and a grant of Korea University Medical Center and Korea University Anam Hospital, Seoul, Republic of Korea (O1700021).

\section{REFERENCES}

1. Neurath MF, Finotto S, Glimcher LH. The role of Th1/ Th2 polarization in mucosal immunity. Nat Med 2002; 8:567-573.

2. Paul WE, Zhu J. How are $\mathrm{T}(\mathrm{H})$ 2-type immune responses initiated and amplified? Nat Rev Immunol 2010;10:225235 .

3. Zhu J, Yamane H, Paul WE. Differentiation of effector $\mathrm{CD}_{4} \mathrm{~T}$ cell populations (*). Annu Rev Immunol 2010;28:445-489.

4. Lambrecht BN, Hammad H. Lung dendritic cells in respiratory viral infection and asthma: from protection to immunopathology. Annu Rev Immunol 2012;30:243-270.

5. Pulendran B, Tang H, Manicassamy S. Programming dendritic cells to induce $\mathrm{T}(\mathrm{H}) 2$ and tolerogenic responses. Nat Immunol 2010;11:647-655.

6. Singh VK, Mehrotra S, Agarwal SS. The paradigm of Th1 and Th2 cytokines: its relevance to autoimmunity and allergy. Immunol Res 1999;20:147-161.

7. Arima M, Fukuda T. Prostaglandin $\mathrm{D}$ and $\mathrm{T}(\mathrm{H}) 2$ inflammation in the pathogenesis of bronchial asthma. Korean
J Intern Med 2011;26:8-18.

8. Kidd P. Th1/Th2 balance: the hypothesis, its limitations, and implications for health and disease. Altern Med Rev 2003;8:223-246.

9. Khodoun MV, Orekhova T, Potter C, Morris S, Finkelman FD. Basophils initiate IL-4 production during a memory T-dependent response. J Exp Med 2004;200:857-870.

10. Tang H, Cao W, Kasturi SP, et al. The T helper type 2 response to cysteine proteases requires dendritic cellbasophil cooperation via ROS-mediated signaling. Nat Immunol 2010;11:608-617.

11. Hammad H, Plantinga M, Deswarte K, et al. Inflammatory dendritic cells (not basophils) are necessary and sufficient for induction of Th2 immunity to inhaled house dust mite allergen. J Exp Med 2010;207:2097-2111.

12. Min B. Basophils induce Th2 immunity: is this final answer? Virulence 2010;1:399-401.

13. Wynn TA. Basophils trump dendritic cells as APCs for $\mathrm{T}(\mathrm{H}) 2$ responses. Nat Immunol 2009;10:679-681.

14. McKenzie AN, Spits H, Eberl G. Innate lymphoid cells in inflammation and immunity. Immunity 2014;41:366-374.

15. Halim TY, Krauss RH, Sun AC, Takei F. Lung natural helper cells are a critical source of Th2 cell-type cytokines in protease allergen-induced airway inflammation. Immunity 2012;36:451-463.

16. Price AE, Liang HE, Sullivan BM, et al. Systemically dispersed innate IL-13-expressing cells in type 2 immunity. Proc Natl Acad Sci U S A 2010;107:11489-11494.

17. Halim TY, Hwang YY, Scanlon ST, et al. Group 2 innate lymphoid cells license dendritic cells to potentiate memory TH2 cell responses. Nat Immunol 2016;17:57-64.

18. Halim TY, Steer CA, Matha L, et al. Group 2 innate lymphoid cells are critical for the initiation of adaptive $\mathrm{T}$ helper 2 cell-mediated allergic lung inflammation. Immunity 2014;40:425-435.

19. Hammad H, Lambrecht BN. Dendritic cells and epithelial cells: linking innate and adaptive immunity in asthma. Nat Rev Immunol 2008;8:193-204.

20. Hammad H, Lambrecht BN. Dendritic cells and airway epithelial cells at the interface between innate and adaptive immune responses. Allergy 2011;66:579-587.

21. Lee HJ, Kim B, Im NR, et al. Decreased expression of Ecadherin and ZO-1 in the nasal mucosa of patients with allergic rhinitis: altered regulation of E-cadherin by IL-4, IL-5, and TNF-alpha. Am J Rhinol Allergy 2016;30:173-178.

22. Soumelis V, Reche PA, Kanzler H, et al. Human epithelial 
cells trigger dendritic cell mediated allergic inflammation by producing TSLP. Nat Immunol 2002;3:673-680.

23. Shortman K, Liu YJ. Mouse and human dendritic cell subtypes. Nat Rev Immunol 2002;2:151-161.

24. Maldonado-Lopez R, De Smedt T, Michel P, et al. CD8alphat and CD8alpha- subclasses of dendritic cells direct the development of distinct $\mathrm{T}$ helper cells in vivo. J Exp Med 1999;189:587-592.

25. Pulendran B, Smith JL, Caspary G, et al. Distinct dendritic cell subsets differentially regulate the class of immune response in vivo. Proc Natl Acad Sci U S A 1999;96:10361041.

26. Lambrecht BN, De Veerman M, Coyle AJ, GutierrezRamos JC, Thielemans K, Pauwels RA. Myeloid dendritic cells induce Th2 responses to inhaled antigen, leading to eosinophilic airway inflammation. J Clin Invest 2000;106:551-559.

27. Stumbles PA, Thomas JA, Pimm CL, et al. Resting respiratory tract dendritic cells preferentially stimulate $\mathrm{T}$ helper cell type 2 (Th2) responses and require obligatory cytokine signals for induction of Thı immunity. J Exp Med 1998;188:2019-2031.

28. Boonstra A, Asselin-Paturel C, Gilliet M, et al. Flexibility of mouse classical and plasmacytoid-derived dendritic cells in directing $\mathrm{T}$ helper type 1 and 2 cell development: dependency on antigen dose and differential Toll-like receptor ligation. J Exp Med 2003;197:101-109.

29. Gao Y, Nish SA, Jiang R, et al. Control of T helper 2 responses by transcription factor IRF4-dependent dendritic cells. Immunity 2013;39:722-732.

30. Kumamoto Y, Linehan M, Weinstein JS, Laidlaw BJ, Craft JE, Iwasaki A. CD3orb dermal dendritic cells drive $\mathrm{T}$ helper 2 cell-mediated immunity. Immunity 2013;39:733743 .

31. Tanaka H, Demeure CE, Rubio M, Delespesse G, Sarfati M. Human monocyte-derived dendritic cells induce naive $\mathrm{T}$ cell differentiation into $\mathrm{T}$ helper cell type 2 (Th2) or Th1/ Th2 effectors. Role of stimulator/responder ratio. J Exp Med 2000;192:405-412.

32. Pulendran B, Banchereau J, Maraskovsky E, Maliszewski C. Modulating the immune response with dendritic cells and their growth factors. Trends Immunol 2001;22:41-47.

33. Rissoan MC, Soumelis V, Kadowaki N, et al. Reciprocal control of $\mathrm{T}$ helper cell and dendritic cell differentiation. Science 1999;283:1183-1186.

34. Klechevsky E, Morita R, Liu M, et al. Functional spe- cializations of human epidermal Langerhans cells and CD14+ dermal dendritic cells. Immunity 2008;29:497-510.

35. Iwasaki A, Medzhitov R. Regulation of adaptive immunity by the innate immune system. Science 2010;327:291-295.

36. Kapsenberg ML. Dendritic-cell control of pathogendriven T-cell polarization. Nat Rev Immunol 2003;3:984993 .

37. Pulendran B, Kumar P, Cutler CW, Mohamadzadeh M, Van Dyke T, Banchereau J. Lipopolysaccharides from distinct pathogens induce different classes of immune responses in vivo. J Immunol 2001;167:5067-5076.

38. Kiura K, Kataoka H, Yasuda M, Inoue N, Shibata K. The diacylated lipopeptide FSL-1 induces TLR2-mediated Th2 responses. FEMS Immunol Med Microbiol 2006;48:44-55.

39. Dillon S, Agrawal A, Van Dyke T, et al. A Toll-like receptor 2 ligand stimulates Th2 responses in vivo, via induction of extracellular signal-regulated kinase mitogen-activated protein kinase and c-Fos in dendritic cells. J Immunol 2004;172:4733-4743.

40. Agrawal S, Agrawal A, Doughty B, et al. Cutting edge: different Toll-like receptor agonists instruct dendritic cells to induce distinct Th responses via differential modulation of extracellular signal-regulated kinasemitogen-activated protein kinase and c-Fos. J Immunol 2003;171:4984-4989.

41. Redecke V, Hacker H, Datta SK, et al. Cutting edge: activation of Toll-like receptor 2 induces a Th2 immune response and promotes experimental asthma. J Immunol 2004;172:2739-2743.

42. van Riet E, Everts B, Retra K, et al. Combined TLR2 and TLR4 ligation in the context of bacterial or helminth extracts in human monocyte derived dendritic cells: molecular correlates for Th1/Th2 polarization. BMC Immunol 2009;10:9.

43. Liu T, He SH, Zheng PY, Zhang TY, Wang BQ, Yang PC. Staphylococcal enterotoxin B increases TIM4 expression in human dendritic cells that drives naive $\mathrm{CD}_{4} \mathrm{~T}$ cells to differentiate into Th2 cells. Mol Immunol 2007;44:35803587 .

44. Yang D, Chen $\mathrm{Q}, \mathrm{Su} \mathrm{SB}$, et al. Eosinophil-derived neurotoxin acts as an alarmin to activate the TLR2-MyD88 signal pathway in dendritic cells and enhances Th2 immune responses. J Exp Med 2008;205:79-90.

45. Eisenbarth SC, Piggott DA, Huleatt JW, Visintin I, Herrick CA, Bottomly K. Lipopolysaccharide-enhanced, toll-like receptor 4 -dependent $\mathrm{T}$ helper cell type 2 responses to 
inhaled antigen. J Exp Med 2002;196:1645-1651.

46. Geijtenbeek TB, Gringhuis SI. Signalling through C-type lectin receptors: shaping immune responses. Nat Rev Immunol 2009;9:465-479.

47. Bergman MP, Engering A, Smits HH, et al. Helicobacter pylori modulates the $\mathrm{T}$ helper cell $1 / \mathrm{T}$ helper cell 2 balance through phase-variable interaction between lipopolysaccharide and DC-SIGN. J Exp Med 2004;200:979990.

48. LeibundGut-Landmann S, Gross O, Robinson MJ, et al. Syk- and CARD9-dependent coupling of innate immunity to the induction of $\mathrm{T}$ helper cells that produce interleukin 17. Nat Immunol 2007;8:630-638.

49. Rogers NC, Slack EC, Edwards AD, et al. Syk-dependent cytokine induction by Dectin-1 reveals a novel pattern recognition pathway for $\mathrm{C}$ type lectins. Immunity 2005;22:507-517.

50. Shreffler WG, Castro RR, Kucuk ZY, et al. The major glycoprotein allergen from Arachis hypogaea, Ara $\mathrm{h}$ 1, is a ligand of dendritic cell-specific ICAM-grabbing nonintegrin and acts as a Th2 adjuvant in vitro. J Immunol 2006;177:3677-3685.

51. Swamy M, Jamora C, Havran W, Hayday A. Epithelial decision makers: in search of the 'epimmunome'. Nat Immunol 2010;11:656-665.

52. Besnard AG, Togbe D, Guillou N, Erard F, Quesniaux V, Ryffel B. IL-33-activated dendritic cells are critical for allergic airway inflammation. Eur J Immunol 2011;41:16751686.

53. Eiwegger T, Akdis CA. IL-33 links tissue cells, dendritic cells and Th2 cell development in a mouse model of asthma. Eur J Immunol 2011;41:1535-1538.

54. Wang YH, Angkasekwinai P, Lu N, et al. IL-25 augments type 2 immune responses by enhancing the expansion and functions of TSLP-DC-activated Th2 memory cells. J Exp Med 2007;204:1837-1847.

55. Yi AK, Yoon JG, Yeo SJ, Hong SC, English BK, Krieg AM. Role of mitogen-activated protein kinases in $\mathrm{CpG}$ DNA-mediated IL-10 and IL-12 production: central role of extracellular signal-regulated kinase in the negative feedback loop of the CpG DNA-mediated Thı response. J Immunol 2002;168:4711-4720.

56. Arima K, Watanabe N, Hanabuchi S, Chang M, Sun SC, Liu YJ. Distinct signal codes generate dendritic cell functional plasticity. Sci Signal 2010;3:ra4.

57. Artis D, Kane CM, Fiore J, et al. Dendritic cell-intrinsic expression of NF-kappa $\mathrm{B} 1$ is required to promote optimal Th2 cell differentiation. J Immunol 2005;174:71547159.

58. Thomas PG, Carter MR, Da'dara AA, DeSimone TM, Harn DA. A helminth glycan induces APC maturation via alternative NF-kappa B activation independent of I kappa B alpha degradation. J Immunol 2005;175:2082-2090.

59. Lee J, Kim TH, Murray F, et al. Cyclic AMP concentrations in dendritic cells induce and regulate Th2 immunity and allergic asthma. Proc Natl Acad Sci U S A 2015;112:15291534 .

6o. Datta SK, Sabet M, Nguyen KP, et al. Mucosal adjuvant activity of cholera toxin requires Th17 cells and protects against inhalation anthrax. Proc Natl Acad Sci U S A 2010;107:10638-10643. 